\title{
Spontaneous and Deflected Drift-Trajectories in Orthogonal Acceleration Time-of-Flight Mass Spectrometry
}

\author{
M. Guilhaus \\ Department of Analytical Chemistry, The University of New South Wales, Sydney, Australia
}

\begin{abstract}
Orthogonal acceleration is a method for gating ions from an ion beam into a time-of-flight (TOF) mass spectrometer. The technique involves a pulsed electric field to apply acceleration directed orthogonally to an ion beam. This approach is useful for coupling continuous ion sources to TOF mass analyzers. Most instruments of this type, which have been described in the literature, use steering electrodes after the orthogonal acceleration step. Those velocity components of ions originating from the ion beam velocity are minimized so that the deflected drift-trajectory is parallel to a transverse flight tube. In an alternative geometry the ion beam velocity is conserved and the drift-trajectory after the orthogonal acceleration step is spontaneous. The differences between the space-time focusing ability with spontaneous and deflected drift-trajectories are discussed and investigated. Trajectory calculations indicate that deflection fields placed after the orthogonal acceleration step distort the ion packet because, in this geometry, the flight-time to the detector is dependent on the position that the ions enter the steering optics. Increasing the duty-cycle efficiency by sampling longer sections of the continuous ion beam leads to a degradation of resolving power. Employing a spontaneous drift-trajectory after orthogonal acceleration provides the advantage that the arrival time spread for isobaric ions is, in principle, independent of the length of the ion beam sampled. The major implication of these findings is that simultaneously optimized sensitivity and resolving power may not be achievable with the deflected drift-trajectory instruments. The calculations are in agreement with results from the published data of a number of groups who have built instruments based on the orthogonal acceleration principle. (I Am Soc Mass Spectrom 1994, 5, 588-595)
\end{abstract}

$\mathrm{T}$ The renaissance of time-of-flight mass spectrometry (TOF/MS) is well underway as evidenced by the large number of articles now appearing in this area. To a large degree the resurgence of interest has been sparked by applications in the biological sciences with pulsed laser desorption methods and ion mirror devices playing major roles $[1,2]$. Significant advances in the enabling technologies of fast computers and timing electronics have also made the engineering of TOF/MS instruments more practical over the last decade.

The use of time-of-flight (TOF) mass analyzers with continuous ion sources, in particular those where the ions are formed in the gas phase, has received considerably less attention than applications with pulsed ion sources. This is largely due to the furdamental difficulties of reducing the effects of initial velocity, position, and time dispersions in these applications [3]. These problems are compounded when the ion source

Address reprint requests to Dr. Guilhaus, Department of Analytical Chemistry, The University of New South Wales, Sydney, 2052, Australia. operates at atmospheric pressure. The dispersions limit the wider application of TOF/MS in the mainstream of mass spectrometry where most ion sources are gaseous and continuous. A device first described by Wollnik and co-workers [4] represents a good solution for coupling TOF/MS to an electron ionization source but this method has not yet been shown to he generally adaptable to a range of ion sources.

The use of orthogonal gating of ions from an ion beam was described recently as a general solution to the problem of coupling TOF/MS to continuous ion sources $[5,6]$. In the earlier publication the label "orthogonal acceleration" was attached to the method and, judging from the titles and abstracts of papers at the 41st Annual ASMS Conference on Mass Spectrometry and Allied Topics, San Francisco, May 30-Jume 4, 1993, it now appears that the name is being accepted and that the orthogonal gating approach is being applied by several groups. In all the applications of orthogonal acceleration TOF/MS, it is acknowledged that the significantly reduced velocity components orthogonal to the continuous beam of ions leads to improved resolving powers compared to the direct 
coupling of TOF /MS with gaseous ion sources in which ion populations are subject to an isotropic MaxwellBoltzmann distribution of initial velocities.

Temporal dispersions are minimal in orthogonal acceleration TOF/MS because the ions, having been formed prior to gating, begin their acceleration simultaneously at the onset of the "push-out pulse" [5]. While a fast rise time of the pulse is desirable, it is the reproducibility of the rising edge of the pulse that is of greater consequence in determining resolving power.

As the velocity and temporal dispersions are well addressed in the method, the spatial dispersion can be corrected with classical spatial focusing [3]. Stated simply, isobaric ions from the back of the ion beam (initially further from the detector) experience more orthogonal acceleration than ions from the front of the beam (initially closer to the detector). The former can be made to catch up to the latter when they reach the detector.

Important in all orthogonal acceleration instruments is the opportunity to attain high duty-cycle efficiencies (sensitivity) when the ratio of axial and orthogonal velocities (after gating) results in the fill-up time of the orthogonal accelerator being of the same order as the drift time to the detector. Thus a relatively long section of the ion beam is accelerated towards the detector.

The history of the orthogonal gating in TOF/MS has not yet been comprehensively cited. The method was used in the nineteen-fifties to study ions formed in the interaction of ionizing electrons with cold molecular beams [7]. In the nineteen-sixties, work done by the Bendix Corporation under defense contracts in the U.S. produced an orthogonal acceleration TOF/MS instrument with an atmospheric-pressure plasma ion-source [8]. An application with this instrument (or a similar one) has been reported in the combustion literature [9]. An instrument with orthogonal gating and an ion mirror was described in a Soviet patent [10] and this work is apparently preliminary to that presented by Dodonov and co-workers in 1991 [11] and related to another Soviet patent (private communication, A. Verentchikov, Physics Department, University of Manitoba, Winnipeg, Canada R3T 2N2) [12]. Recently the geometry of the Bendix instrument has been used for a variety of atmospheric pressure ion sources [13-15].

While the method of orthogonal acceleration is a useful way to create packets of ions for TOF mass analysis, the arrangement of the mass analyzer after the orthogonal acceleration step has been approached in a varicty of ways involving combinations of deflection plates and ion mirrors. A key distinction in the ion optics is that they produce either a transverse $\left(90^{\circ}\right)$ or oblique $\left(3^{\circ}-10^{\circ}\right.$ from perpendicular) drift-trajectory. The differences in these geometries may appear superficially small but careful consideration of the effect of, for example, post-acceleration deflection fields, suggest that significant differences in the ability of the optics to achieve space-time focusing may exist amongst the geometries. In regard to a deflected drift-trajectory instrument, Lee [14] has stated that "... the best resolution was obtained with no deflection voltage applied...," however, no explanation was given for this phenomenon. It is the effect of the deflection plates on resolving power that is the principal theme of this article.

Net applied forces and ion trajectories in orthogonal acceleration instruments clearly distinguish three different approaches, leaving aside, for the moment, the presence or absence of an ion mirror. Figure 1 shows three orthogonal acceleration geometries. It is convenient to define the direction of the continuous ion beam as the axial direction $(x)$ such that the pulsed acceleration is applied orthogonally to it $(y)$.

\section{A. Orthogonal Acceleration-Deflected Drift- Trajectory (Axial Retardation)}

This geometry is used in the Bendix apparatus [8] and a number of more recent instruments $[9,13-16]$. The deflection plates in this method produce a field that imparts an axial deceleration to the ions that have been orthogonally gated. The orthogonal component of the net deflection force acts to increase the ion velocity in that direction. In fact, it is a simple consequence of the conservation of.linear momentum that this must be the case if the potential energy of the ions, before and after deflection, is unchanged. The deflection plates, being akin to a small electric sector, will be energy dispersive and thus the distribution of trajectory directions after steering will be influenced by the kinetic energy distribution of the ions. The electric field created by steering plates in the ideal case would be homogeneous and perpendicular to the orthogonal acceleration field. As will be discussed in more detail later, even in this ideal case the ions that travel over the high potential part of the field have a lower velocity there while the ions in the low potential zone have a higher velocity. The difference in flight-times in these regions brings about a rotation of an ion packet.

In practice, electrodes cannot be arranged to linearize the field (these would block the passage of the ions). The fringing fields and field inhomogeneities are therefore significant and will affect the arrival-time spread of isobaric ions at the detector. The deflection of the ions thus affects their velocities in the TOF direction; it is not clear that space-time focusing can be preserved. Even though the deflection angles, hence velocity changes, are small, time differences of $1 \mathrm{~ns}$ in $100 \mu$ s (i.e., 1 in $10^{5}$ ) are significant in determining the resolving power of a medium resolution TOF mass analyzer.

\section{B. Orthogonal Acceleration-Spontaneous Drift- Trajectory}

This geometry conserves the axial velocity of the ions and produces a spontaneous drift-trajectory, which is 
obliquely directed. The ions are subject only to the net force from the orthogonal acceleration. If the orthogonal velocity spread of ions in the beam is minimal, the spatial spread can be corrected to a large extent. This approach was originally described by Dawson and Guilhaus [5] with only two other reports of this geometry apparent to the author of this paper [11, 17]. It is

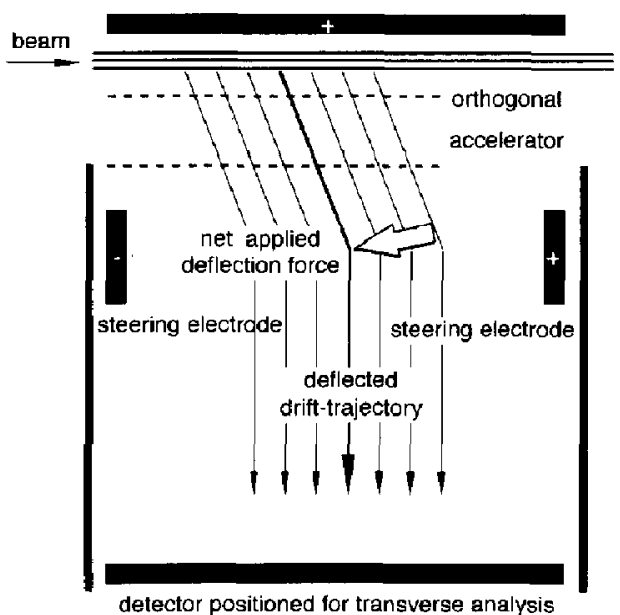

a

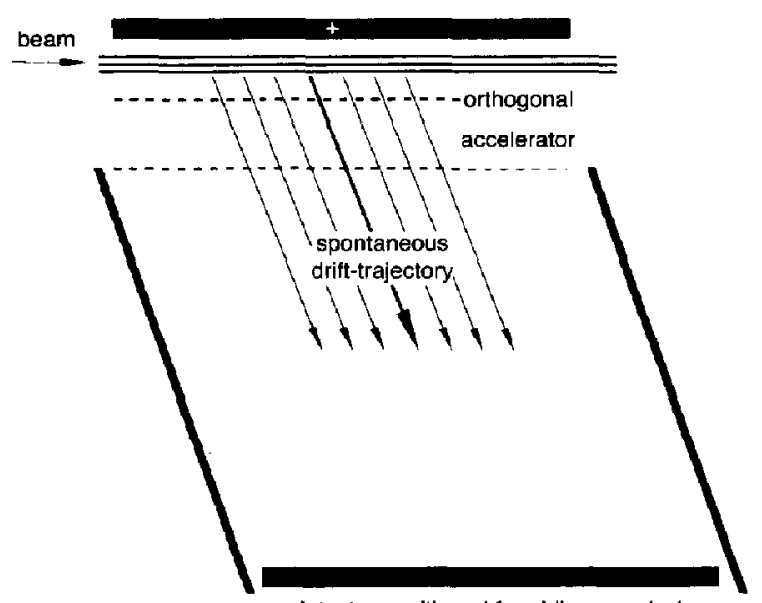

detector positioned for oblique analysis

b

Figure 1. Net deflection forces and ion trajectories in orthogonal acceleration TOF/MS instrument geometries: (a) deflected drifttrajectory with axial deceleration; (b), spontaneous drift-trajectory; and (c), deflected drift-trajectory with axial acceleration. In this classification it is accepted that the spontaneous drift-trajectory geometry encompasses the use of deflection plates to facilitate slight adjustment of the oblique trajectory should it be necessary, for example, to compensate for small changes in the energy of the continuous ion beam. In such cases the deleterious effects of the deflection are also slight.

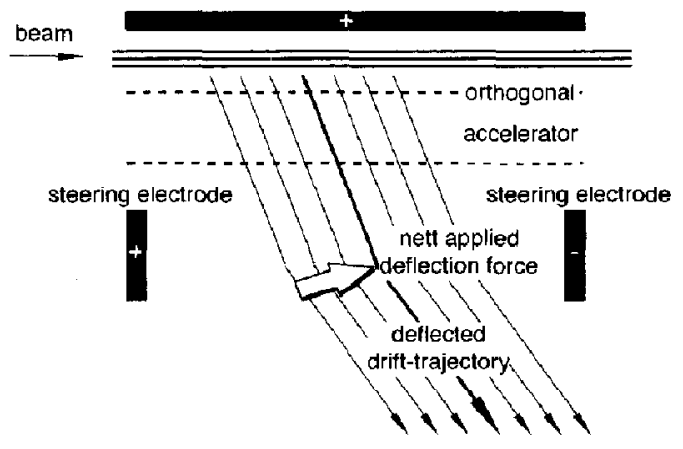

to ion mirror

c

Figure 1. (Continued)

noteworthy that a related TOF approach involving the conservation of axial velocity together with a positionsensitive detector has been used to provide data on the energy distribution of ions in molecular beams [18].

\section{Orthogonal Acceleration-Deflected Drift- Trajectory (Axial Acceleration)}

This geometry involves deflection plates which impart axial acceleration to the ion packet after orthogonal gating. The apparent reason for this step is to direct the trajectory of the ions into an ion mirror such that sufficient displacement between orthogonal accelerator and detector can be realized. Only one reference to this geometry has been found [10] and a detailed discussion of the geometry will nut be given here.

The complex influence of the deflection fields in Geometry $A$ would be expected to change the spacetime focusing characteristics of the TOF mass analyzer. The magnitude of the effect of these factors is by no means obvious. In order to provide an initial assessment of this problem, a set of representative ion-trajectory calculations was performed in which various deflection fields were modeled accurately. The space-time focusing properties of the geometry were compared with a corresponding spontaneous drift-trajectory geometry.

\section{Methods}

The effect of steering ion packets for orthogonal acceleration with deflected drift-trajectories has been invesligated by adding, in simulation, deflection plates after the orthogonal accelerator of the instrument of Ref. 5. Three deflection plate geometries with respectively length-to-separation ratios and applied potential differences of $0.41,1004 \mathrm{~V}$ (II); 1.0, 411 (III), and 2.0, 242 V (IV) were chosen. Simulations were performed with a program called I-Opt, which has been briefly de- 
scribed elsewhere [5]. I-Opt is similar to well-known packages like SIMION but differs in its ability to handle much larger arrays (typically 100,000 elements). The trajectory algorithms in I-Opt are optimized for accuracy of TOF and final velocity data.

The cross-sectional layout of an orthogonal acceleration device $[5,6]$ with added axial deflection electrodes (II) is shown in Figures 2 and 3. Figure 2 shows the potential function resulting from the numerical solution of the Laplace Equation. It should be noted that two symmetries were required to represent the combined devices for orthogonal acceleration (cylindrical symmetry) and post-orthogonal-acceleration deflection (planar symmetry). Figure 3 shows the trajectories of orthogonally accelerated and axially decelerated ions in (II). The data were obtained by performing two simulations of ion trajectories, passing the final parameters of the first (orthogonal acceleration) to the initial parameters for the second (post-orthogonal-acceleration deflection). Trajectories were calculated for ions originating in the parallel ion beam with a kinetic energy of $14.0 \mathrm{eV}$ (with no initial orthogonal velocity component and no spread in axial velocities). Ions were generated at various positions in the beam or at the entrance to the steering optics. Time-resolved trajectories were generated from I-Opt to determine the effect of the steering field. The final kinetic parameters for the ions were projected numerically through the field-free drift region to a specific length in the driftlength/time-plane where the arrival times of the ions were most closely bunched. This indicated the optimum drift-region length and the spread of arrival times. The whole process was repeated without any

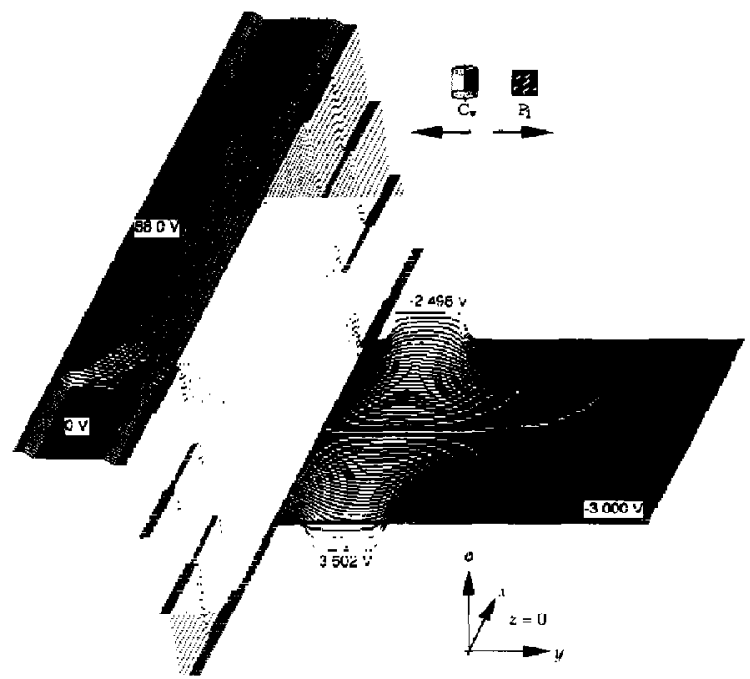

Figure 2. Three-dimensional representation of the potential function in the layout of Figure 3. The potentials are for the state in which the push-out potential has been applied to create the first stage of orthogonal acceleration $[5,6]$. Images were produced with a software simulation package, 1-Opt [5].

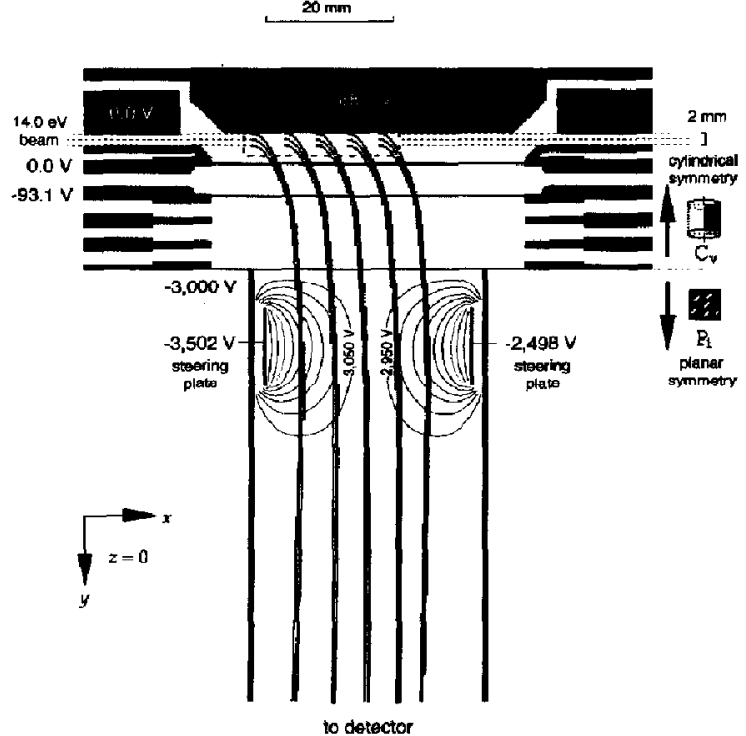

Figure 3. Cross-sectional layout of electrodes in the orthogonal acceleration TOF/MS instrument of Refs. 5 and 6 (cylindrical symmetry) with added deflection plates (planar symmetry) producing a Geometry $A$ (II) configuration. Fifteen trajectories are shown from initial positions in a 20 -rmm section of the $2 \mathrm{~mm}$-wide beam. Equipotentials at $50.0 \mathrm{~V}$ intervals are shown in the vicinity of the steering electrodes. Calculations were performed with a software simulation package called I-Opt which has been described elscwhere [5].

axial deceleration device to obtain comparative data for orthogonal acceleration/spontaneous drift-trajectory geometry.

\section{Results and Discussion}

The trajectory simulation package used in this work has the useful ability to display time-resolved trajectories. The position of ions in space and at regular intervals in time can be seen simultaneously, as shown in Figure 4. The dotted lines are ion trajectories for ions ( $m / z 132$ ) entering the steering optics from the final acceleration grid (see Figure 3). The ions enter this simulation with kinetic energy corresponding to ions at the same grid that originated along the axis of the continuous ion beam, as shown in Figure 3. Time contours are shown at $50 \mathrm{~ns}$ intervals. Two significant observations can be made from this calculation:

1. The time contours are no longer parallel to the grid after passing through the steering optics-the ions entering at the right of the optics are lagging behind those entering at the left.

2. The ions entering at the left and right of the center of the steering optics leave with trajectories drifting to the left. 
The observations can be explained readily, As illustrated by Figure 2, the ions that travel over the high potential part of the deflection field are decelerated (in y) and have a lower velocity (in y) whilst in that region. The converse applies to ions which travel via the low potential part of the field. The former will therefore lag behind the latter bringing about a rotation of the parket, as shown in Figure 4.

Due to the nonlinearity of the deflection field, the field will always be stronger nearer the plates (see equipotentials in Figure 3). Thus ions passing nearer the plates will be deflected more strongly than ions in the middle.

These obscrvations raise the question of whether this perturbation of the ion packet can be corrected. For example, a rotation of the detector plane may correct much of the arrival time-spread if the relationship between the position of the ions (in the $x$-direction) and their time-lag is preserved during the TOF to the detector. The effect of the lateral drift of the trajectories entering at either extreme of the optics will be to bring these ions to the detector further to the left. This may preclude correction of the arrival time spread by rotation of the detector.

In each case (II-IV) 15 trajectories of ions from a 20-mm length of the ion beam were calculated to the end of the ion optics, as shown in Figure 3. The final temporal, kinetic, and spatial parameters of these ions were used to locate that drift-region length at which the ion arrival-time spread was minimized. The position of each of the ions was then calculated at the time that the first ion in the packet arrived at a detector located at that length. This is shown in Figure 5 with

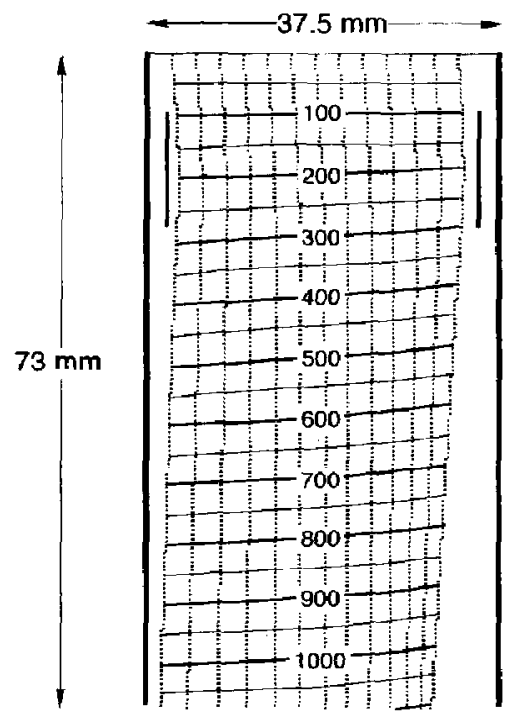

Figure 4. Time-resolved trajectories for ions entering the deflection optics (II) with the kinetic parameters of ions which would have originated along the axis of the continuous ion beam. The time contours are at 50 ns intervals and labeled in ns. the results from a similar treatment of ions passing through a spontaneous drift-trajectory apparatus (I). Figure 6 shows the total arrival-time spread as a function of drift-length for all geometries.

Figures 5 and 6 reveal the distortion inflicted on the ion packet by the deflection optics. The following observations are significant:

1. The optimum detection plane is moved to a longer drift-length than for the spontaneous drift-trajectory case.

2. The spatial focusing for groups of ions originating from the same position along the ion beam $(a-e)$ is slightly perturbed for ions not passing through the center of the steering optics.

3. Ions arrive over a larger $x$-distance in the deflected drift-trajectory case and a larger detector would be required; the effect is greater for the shortest deflection plate geometry (II).

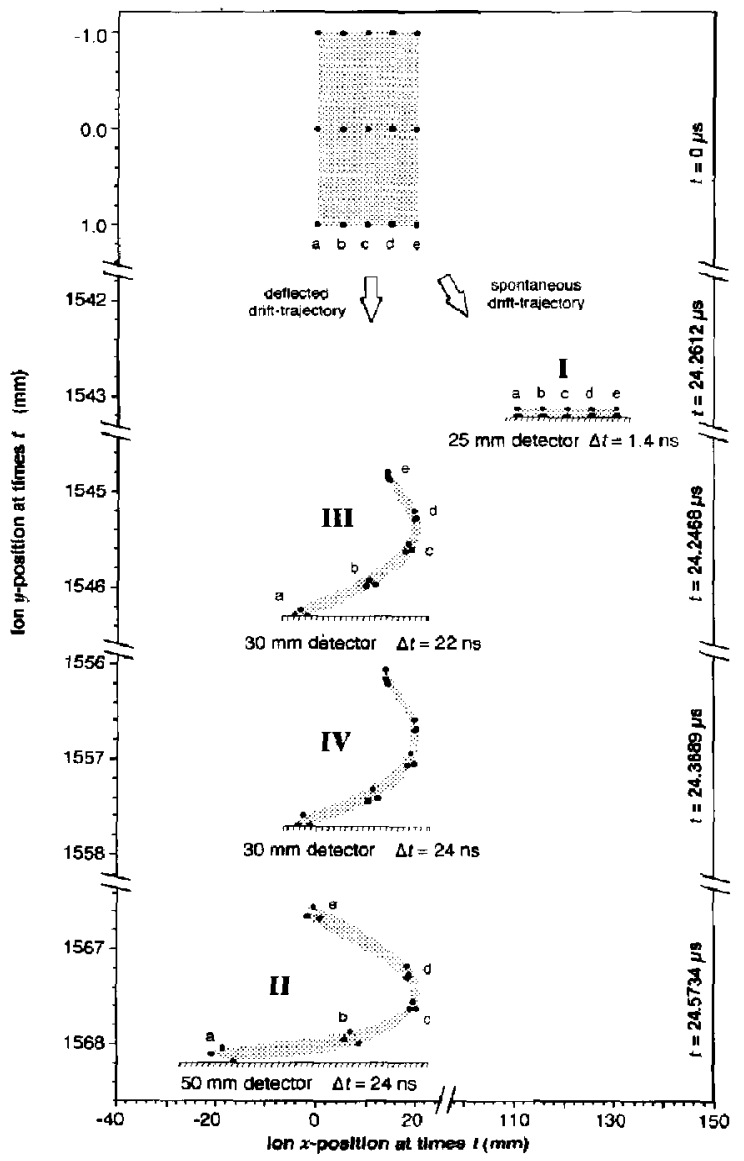

Figure 5. Mapping of ion packet from its origin in the ion beam $(t=0, y=-1,-0,1 \mathrm{~mm}, x=0,5,10,15,20 \mathrm{~mm})$ to the detector for deflected (II)-(IV) and spontaneous (I) drift-trajectories. 


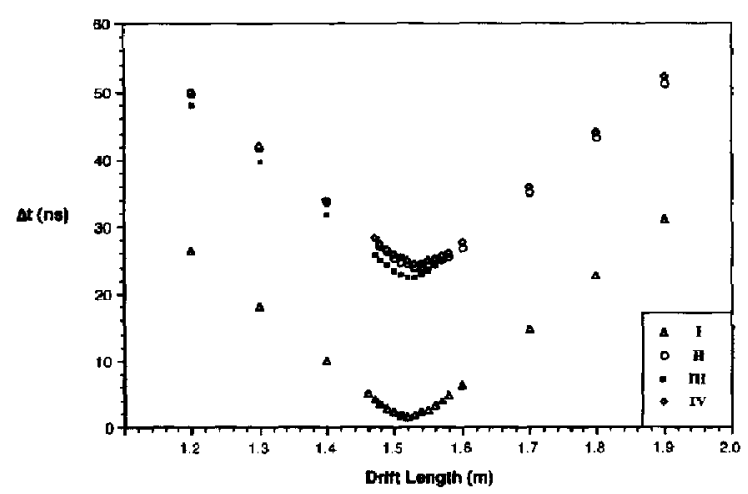

Figure 6. Arrival time spread $\Delta t$ as a function of the drift-length for the deflected drift-trajectory geometries (II)-(IV) and the spontaneous drift-trajectory geometry (I). $\Delta t$ is the difference in the arrival times of the first and last of the fifteen ions (Figure 3) to reach the detector for each of the configurations investigated.

4. The deflected ion packets fold over during the TOF to the detector giving rise to substantial arrival-time spreads (22-24 ns) relative to the time-spread due to spatial focusing in the spontaneous drift-trajectory case ( $1.4 \mathrm{~ns})$.

5. The optimum drift-length is determined by the spatial focusing. Although a reduction of $\Delta t$ from the distortion of the ion packet will be effected by shortening the drift-length, the corresponding increase in $\Delta t$ from the spatial spread is significantly greater.

6. The longer deflection plate geometries (III, IV) certainly improve the geometric focusing of the spectrometer (ions can be collected on a smaller detector), but there is no significant reduction in the arrival-time spread, $\Delta t$.

It is interesting that, compared to the short geometry (II) the longer deflection plates improve geometric focusing of the beam but do not significantly improve the time-focusing or the packet distortion. Lengthening the deflection plates reduces the potential difference needed to steer the ion packet and thus, also, the velocity difference of the ions on either side of the packet as it passes between the plates. The increase in length, however, necessitates a longer transit time. Neglecting field nonlinearity for a moment, the smaller velocity difference for ions traveling between the longer plates is largely canceled by the longer transit time (about the same difference in transit-time accumulates).

Now, considering the nonlinearity of the deflection field, as mentioned above, ions passing nearer the plates will be deflected more strongly than ions in the middle. This gives rise to the stretching and folding over of the packet as it drifts to the detector. The effect is eased, but not dramatically, by lengthening the geometry as seen in Figure 5. Here the increased length brings a longer exposure to a reduced nonlinearity. The effects cancel (at least partially) to preserve a large degree of packet distortion.

These results suggest that increased ion arrival-time spread from the distortion of the ion packet caused by the steering optics cannot be easily corrected. The arrival-time spread could be reduced by accepting only ions from a short length of the ion beam (relative to the separation of the steering electrodes). This measure would significantly reduce the efficiency of the duty-cycle of the apparatus. It is noteworthy that the apparatus described by Bergman [13] obtains very high resolutions but with considerable limitations on the volume of the beam from which ions can be sampled.

These results show that the deliberately engineered and desirable wide ion packet in orthogonal acceleration TOF/MS is much more sensitive to the degradation of resolution by deflection than a narrow packet as produced in an in-line geometry. However, as advocated by this author, the deflection step should be avoided whereupon significant duty-cycle and resolution gains over most in-line approaches are attainable.

This analysis has neglected a number of important sources of arrival-time spread in orthogonal acceleration instruments. Initial ion velocity orthogonal to the beam has been set to zero and the lens effects of meshes [5, 19] have not been considered. Of lesser importance, but still worthy of mention, is the instability of the accelerating high voltage. The finite detector-pulse width and broadening of it by the bandwidth limit of the electronics can, in high resolution TOF instruments, be a significant contributor to peak width at low mass [21].

The current density across the collimated ion beam of the author's apparatus has been measured $450 \mathrm{~mm}$ beyond the $2 \mathrm{~mm}$ exit aperture of the collimation lens. A spatial distribution approximately characterized by a standard deviation of $0.4 \mathrm{~mm}$ was evident at this beam position. The ions reach this position without encountering any additional apertures or focusing optics. From this datum an upper limit in the standard deviation of the orthogonal velocity distribution $\left(\sigma_{v \varphi}\right)$ of the $14 \mathrm{eV}{ }^{132} \mathrm{Xe}$ ions $\left(x_{x}=4500 \mathrm{~ms}^{-1}\right)$ can be estimated. If one considers the worst case of an ion trajectory from an edge of the collimation lens exit aperture to one spatial-distribution standard deviation on the opposite side of the beam axis and $450 \mathrm{~mm}$ away:

$$
\begin{aligned}
\sigma_{v y}(\max ) & =1.4 / 450 \times 4500 \mathrm{~ms}^{-1} \\
& =14 \mathrm{~ms}^{-1}
\end{aligned}
$$

From peak shape modeling (a description of which will be published separately), it is apparent that a $\sigma_{v y}$ $=12 \mathrm{~ms}^{-1}$, somewhat less than the upper limit, is more likely. This alone would give rise to $2.3 \mathrm{~ns}$ (FWHM) arrival time spread in the author's $3 \mathrm{kV}, 1.5 \mathrm{~m}$ 
instrument. Mesh effects and ripple on the high-voltage supply are estimated to broaden this to $3.4 \mathrm{~ns}$.

The detector pulse width, of $\sim 2$ ns (Galileo type $3025 \mathrm{~N}^{\mathrm{MA}}$ Chevron ${ }^{\mathrm{TM}}$ ), after passing through the 350 $\mathrm{MHz}(3 \mathrm{db}$ ) bandwidth of the LeCroy 9450 is broadened by $20 \%$ to about $2.4 \mathrm{~ns}$ [20]. A method has been developed [21] in the author's laboratory whereby a graph of observed pulse-width versus $/$ mass, extrapolated to $\gamma$ mass $=0$ indicates the combined effect of detector-pulse, bandwidth, and jitter. This method indicates a value of $2.9 \mathrm{~ns}$ which is quite feasible considering that the $2.4 \mathrm{~ns}$ estimate above does not consider jitter in timing and triggering electronics.

The time-spreads are not correlated and should add in root-mean-square fashion to explain the observed $4.5 \mathrm{~ns}$ peak width of the ${ }^{132}$ Xe ion as shown in Table 1. Also presented in Table 1 is the effect of increasing the arrival-time spread due to deflection into an orthogonal drift-trajectory as calculated in this article. The increase in peak width and associated degradation of resolving power is quite large.

At the time of writing this article the author is aware of six recent accounts of orthogonal acceleration instruments in the literature. Of these, three $[6,11 a]$ employ a spontaneous trajectory by the definition stated earlier. The average resolving reported for these instruments is $\sim \mathrm{m} / \Delta \mathrm{m}=4000$. The remaining instruments [14-16] use deflected trajectories into orthogonal drift-tubes and give an average resolving power of $\mathrm{m} / \Delta \mathrm{m}<1000$. This is a large discrepancy, which is in qualitative agreement with the predictions of Table 1. Even though the reports describe instruments with a range of ion sources, drift-lengths, as well as linear and reflector designs, this author proposes that the use of the deflected geometry contributes significantly to a loss of resolving power. Indeed, the electrospray instruments are similar [11b, 16], both employing reflectors yet the spontaneous trajectory configuration $[11 \mathrm{~b}]$ offers a resolving power

Table 1. Differences in full width at half maximum (FWHM) arrival-time spreads (ns) and resolving powers with spontaneous- and deflected-drift-trajectory orthogonal acceleration TOF/MS geometries for $m / \Sigma 132$ where the time separation from $m / z 133$ is 90.0 ins

\begin{tabular}{|c|c|c|c|c|c|}
\hline Geometry & $\begin{array}{c}\text { A } \\
\text { Velocity } \\
\text { and } \\
\text { Meshes }\end{array}$ & $\begin{array}{c}\text { B } \\
\text { Spatial } \\
\text { and } \\
\text { Steering }\end{array}$ & Detection' & Total $^{c}$ & $\mathrm{~m} / \Delta \mathrm{m}$ \\
\hline Spontaneous & 3.4 & 0.1 & 2.9 & $4 . b$ & 2,640 \\
\hline Deflected & 3.4 & 12 & 2.9 & 12.8 & 930 \\
\hline
\end{tabular}

As calculated in this work: the total observed spread was halved to approximate the expected FWHM value. In fact, for the spontaneous trajectory, this contribution would be much less because the unperturbed spatial focusing produces an arrival-time distribution which is very narrow at the FWHM compared to the base.

'Combination of detector pulse (broadened by bandwidth of electronicsl and jitter in electronics.

- The individual contributions are not correlated and so add according to $D^{2}=A^{2}+B^{2}+C^{2}$. over four times that of its deflected trajectory counterpart. It would be fair to say that the evidence to date at least supports the proposition.

\section{Conclusions}

In contrast to the complexity and limitations of deflected drift-trajectory (Geometry $A$ ), the spontaneous post-acceleration drift-trajectory in Geometry $B$ requires no steering field. The oblique direction of the drift-trajectory simply requires relocation of the detector. To date the best resolving powers reported from orthogonal acceleration TOF/MS instruments operating in the long-beam-length extraction mode, have been $\sim \mathrm{m} / \Delta \mathrm{m}(\mathrm{FWHM})=4000-5000[6,11 \mathrm{~b}]$. Both devices employ spontaneous drift-trajectories after the orthogonal acceleration step. Accounts of instruments employing nonspontaneous drift-trajectories after the orthogonal acceleration step report resolving powers in the range $m / \Delta m(F W H M)=450-1200[10,14-16]$. The results of the calculations presented in this paper suggest that the deflecting fields employed in the latter class of instrument are responsible to a significant extent for the lower resolving power.

\section{Acknowledgments}

The author thanks Drs. C. M. Whitehouse and J. G. Boyle, Analytica Inc. (Branford, CT), for bringing Refs. 7 and 8 to his attention. The financial support for this project from the Australian Research Council, the Department of Industry. Technology and Commerce and GBC Scientific Equipment (Dandenong, Australia) is gratefully acknowledged. Thanks are also expressed to Dr. Dmytro Bandura, Dr. Rob Danby, GBC, and Dr. M. J. Lacey, CSIRO division of Entomology, for helpful comments.

\section{References}

1. Cotter, R. J. Anal. Chem. 1992, 64, 1027A-1039A.

2. Cotter, R. J. Biomed, Environ. Mass Spectrom. 1989, 18, 513-532.

3. Sanzone, G. Rev. Sci. Instrum. 1970, 41, 741-742.

4. Grix, R.; Grüner, U.; Li, G.; Stroh, H.; Wollnik, H. Int. I. Mass Spectrom. Ion Processes 1989, 93, 323-330.

5. Dawson, J. H. 1.: Guilhaus, M. Rapid Commun. Mass Spectrom. 1989, 3, 155-159.

6. Coles, J.; Guilhaus, M. Trends Anal Chem. 1993, 12, 203-213.

7. Benson, S. W.; Grossman, J. J.; Elkin, H. S. U.S. Patent No. 2,938,116, May 24, 1960 (filed April 2, 1956).

8. O'Halloran, G. J.; Fluegge, R. A.; Betts, J. F.; Everett, W. L. Technical Documentary Report, No. ASD-TDR-62-644, Part I \& II, prepared under Contract Nos. AF33(616)-8374 and AF33(657)-11018 by The Bendix Corporation, Research Laboratories Division, Southfield, Michigan, 1964 .

9. Gerhardt, Ph,; Löffler, S.; Homann, K. H. Proceedings of the 22nd Symposium (International) on Combustion; The University of Washington; Seattle, WA, August 14-19, 1988, pp. 395-401.

10. Chernushevich, I. V.; Dodonov, A. F.; Dodonova, T. F., Inventors; USSR Chem. Phys. Energy Problems Inst., Assignee. Soviet patent No. SU 1681340 A1, Mass Spectrontetric Analysis According to Transit Time of Continuous Ion Beam-Inoolves Feeding Tons into Modulator in Direction Perpendicular to Axis of Drift Tube (filed February 25, 1987). 
11. (a) Dodonov, A. F.; Chernushevich, I. V; Laiko, V. V. Extended Abstract from the Procedings of the 12th International Mass Spectrometry Conference; Amsterdam, The Netherlands, August 26-30, 1991; (b) see also Verentchikov, A.; Ens, W: Standing, K. G. Paper MOA 10:50 in Abstracts and Proceedings of the 41st Annual ASMS Conference on Mass Spectrometry and Allied Topics; San Francisco, CA, May 30-June 4, 1993.

12. Dodonov, A. F.; Chernushevich, I. V.; Dodonova, T. F.; Raznikov, V. V.; Talroze, V .L., Inventors; USSR Chem. Phys. Energy Problems Inst., Assignee. Soviet patent No. WO 91/03071 (PCT/SU89/00228), Method and Device for Continuous-Waze Iont Beam Time-of-Flight Mass Spectrometric Analysis (filed August 25, 1989).

13. Bergmann, T.; Martin, T. P.; Shaber, H. Rev. Sci. Instrum. 1990, $61,2585-2591$.

14. Sin, C. H.; Lee, E. D.; Lee, M. L. Anal. Chem. 1991, 63, 2897-2900.
15. Myers, D. P; Hieftje, G. M. Proceedings of the 41st Annual ASMS Conference on Mass Spectrometry and Allied Topics; San Francisco, CA, May 30-June 4, 1993, ThP 131.

16. Boyle, J. G.; Whitehouse, C. M. Anal. Chem., 1992, 64, 2084-2089.

17. Clayton, F.; Bateman, R. H. Rapid Comm. Mass Spectrom. 1992, $5,719-720$.

18. Pollard, J. E.; Lichtin, D. A.; Janson, S. W.; Cohen, R. B. Rev. Sci. Instrum. 1989, 60, 3171-3180.

19. Bergmann, T.; Martin, T. P.; Shaber, H. Rev. Sci. Instrum. 1989, 60. 347.

20. Application Note ITI 015B, Characterising and Troubleshooting High Speed Circuits, The LeCroy Corporation: Chestnut Ridge, NY, June 1993, p. 4.

21. Coles, J. N.; Guilhaus, M. J. Am. Soc. Mass Spectrom. 1994, 5, in press. 